\title{
Computational Muscle Driven Knee Simulator for Assessment of Total Knee Replacement Post-Cam Mechanics
}

\author{
Mohd Afzan Mohd Anuar ${ }^{*}$, Mitsugu Todo ${ }^{2}$ \\ ${ }^{I}$ Faculty of Mechanical Engineering, Universiti Teknologi MARA, 40450 Shah Alam, Selangor, Malaysia \\ ${ }^{2}$ Research Institute for Applied Mechanics, Kyushu University, 6-1 Kasuga-koen, Kasuga, Fukuoka, 816- 8580, Japan \\ *Corresponding author E-mail: afzan341@salam.uitm.edu.my
}

\begin{abstract}
Biomechanics of post-cam mechanism is essential in determining the longevity of knee implant. Computational knee simulator is an efficient method in characterizing TKA performance under various boundary conditions. The existing knee simulators, however, were actuated only by quadriceps translation and hip load to perform squatting motion. The present computational knee simulator was developed based on lower limb of Japanese female subject having body weight, $W=51 \mathrm{~kg}$ and height, $H=148 \mathrm{~cm}$. Two different designs of PS-type knee prostheses were tested namely Superflex and NRG. The knee motion was driven by three major muscles; quadriceps, hamstrings and gastrocnemius. The biomechanical behavior of tibiofemoral articulation associated with post-cam engagement mechanics was observed. Post-cam engagement occurred at $80^{\circ}$ and $65^{\circ}$ of flexion angles for Superflex and NRG, respectively. Maximum von Mises stresses at tibial post were $80 \mathrm{MPa}$ and $50 \mathrm{MPa}$ for Superflex and NRG, respectively. The developed computational muscle driven knee simulator has successfully assessed the performance of TKA prostheses.
\end{abstract}

Keywords: Knee simulator; post-cam mechanics; von Mises stress; tibial rotation.

\section{Introduction}

Introduced in the last four decades, posterior-stabilized (PS) TKR procedure was designed to substitute PCL function in governing anterior-posterior (AP) translation and allowing femoral rollback [1]. PS-TKR knee implant is commonly designed to have a tibial spine and femoral cam that interact at certain flexion angle as the knee bends. The biomechanics of the post-cam mechanism are important in determining the longevity of knee implant that partly involve tibial post failure, and post-operative knee kinematics associated with the range of motion (ROM), AP translation and tibial rotation [2]-[3]. As the TKA studies for elder patients were extensively performed, number of TKA mechanics analysis for young and active patients are significantly limited.

Investigations on post-cam mechanics were carried out by various approaches as reported in previous literatures. Most studies assessed the effect of post-cam design on tibiofemoral contact mechanics, tibial post internal stress and kinematics of implanted knee. Some researchers performed contact analysis on knee implant using in vitro testing devices. They measured contact areas and contact stress of various designs at different tibial and flexion angles using stress sensor [4]-[5]. In other studies, computational simulations were implemented to investigate the influence of postcam geometry on contact mechanics and post internal stress [6] [7]. Finite element analysis was performed typically by applying posterior load, compressive load, flexion/extension moment or any combination thereof at various tibiofemoral alignments. Knee kinematics, in part, is also an important outcome of TKA. Numerous studies on tibiofemoral kinematics were done commonly involved in vivo fluoroscopic analysis [3], [8]-[9]. Yet, these studies used static (or quasi-static) approaches by investigating a limited number of static positions of tibiofemoral joint during flexion.
Wear, in part, is a major problem of TKA as reported in previous clinical studies [10]-[12] leads to the TKA implant wear testing using simulator machine. Mechanical wear simulator is a machine designed in such way to apply a large number of cyclic loadings to one or a group of knee implants [13]-[14]. Most wear simulators simulate normal walking gait motions and loadings in a controlled environment with appropriate lubrication. For multi-station simulator, it capable in performing wear testing on a set of knee implants with different designs and properties. However, there are a few limitations such as exclusion of patellofemoral articulations, position driven and omission of knee joint ligamentous effect. Thus, force controlled and muscle driven knee simulator have been developed to address such drawbacks, as reported in previous manuscripts [15]-[16]. TKA experimental evaluations, however, might be difficult, costly and time consuming. Computational simulation has been found to be essential and efficient in characterizing TKA performance under various loadings and boundary conditions. FE models that replicate knee simulator were developed including Kansas knee simulator model [2], [17] and Stanmore knee simulator model [18].

The above mentioned knee simulator models, however, were actuated only by means of quadriceps translation and hip load to perform deep bending motion. Escamilla reported that knee stability during squat is largely contributed not only by quadriceps, but also hamstrings and gastrocnemius muscles [19]. Dahlkvist et al. solved inverse dynamics problem of lower limbs during descent and obtained peak muscle forces of $1640 \mathrm{~N}$ and $1418 \mathrm{~N}$ for hamstrings and gastrocnemius, respectively [20]. Finni et al. measured almost 2 times bodyweight of in vivo Achilles tendon force induced during fast squat [21]. These substantial muscle forces may induce high joint load at tibiofemoral articulations. Other studies reported the significance of hamstrings muscle load on knee kinematics during high flexion [22]. 
In this study, we developed a computational knee simulator that reproduced knee squatting motion by the action of three major muscles; quadriceps, hamstrings and gastrocnemius, to investigate the post-cam mechanics of PS-type knee prostheses.

\section{Methods and Analysis}

The computational knee simulator was developed based on lower limb of Japanese female subject having body weight, $W=51 \mathrm{~kg}$ and height, $H=148 \mathrm{~cm}$ [23]. Mass and inertial properties of each segment including pelvis / upper body segment, femur / thigh segment, patellar, knee prosthesis and tibia / lower leg segment were computed based on Winter (2009) and Hall (2012) as shown in Table 1 [24]-[25].

FE model of simulator implanted with knee prosthesis was developed for dynamic simulation in LS-DYNA (LSTC, Livermore, California, USA). For all components, tetrahedron elements were used with edge length ranged between 1.2 and 12 $\mathrm{mm}$. Polyethylene (PE) insert was represented as non-linear elastic plastic material having initial Young`s Modulus, $E=800 \mathrm{MPa}$ and Poisson`s ratio, $v=0.40$. To reduce computational time, all simulator parts including femoral and tibial component were considered as rigid body. Due to significant variation of elastic bulk modulus between femoral component and tibial insert, soft constraint-based formulation was applied to tibiofemoral contact with coefficient of friction of 0.04 [26]

Table 1: Solid Properties of Each Segment in the Knee Simulator

\begin{tabular}{|c|c|c|}
\hline Segment & Mass (kg) & $\begin{array}{l}\text { Moment of inertia } \\
\left(\mathrm{kg} \cdot \mathrm{mm}^{2}\right)\end{array}$ \\
\hline $\begin{array}{lll}\begin{array}{l}\text { Pelvis / Upper body } \\
\text { segment }\end{array} & \\
\end{array}$ & 16.155 & $I x x=I y y=I z z=1$ \\
\hline Thigh & 6.012 & $\begin{array}{l}I x x=I y y=84350 \\
I z z=1\end{array}$ \\
\hline Femoral component & 0.161 & $\begin{array}{l}I x x=70.355 \\
I x y=-8.0869 \\
I x z=-1.7072 \\
I y y=72.898 \\
I y z=-4.9006 \\
I z z=111.84\end{array}$ \\
\hline Patellar & 0.025 & $I x x=I y y=I z z=0.001$ \\
\hline Tibial component & 0.061 & $\begin{array}{l}I x x=9.7111 \\
I x y=-0.56547 \\
I x z=0.0045933 \\
I y y=20.502 \\
I y z=0.55501 \\
I z z=25.714\end{array}$ \\
\hline Lower leg & 2.738 & $\begin{array}{l}I x x=I y y=23247 \\
I z z=1\end{array}$ \\
\hline
\end{tabular}

The knee joint model included four major ligaments, lateral collateral ligament (LCL), anterior medial collateral ligament (aMCL), oblique medial collateral ligament (oMCL) and deep medial collateral ligament (dMCL) which were inserted based on previous literature [27]. All ligaments were considered as nonlinear springs with the mechanical properties were simulated to implement following force-displacement relationship as in (1).

$$
F= \begin{cases}0 ; & \varepsilon_{i} \leq 0 \\ k_{1}\left(L_{i}-L_{0 i}\right)^{2} ; & 0<\varepsilon_{i} \leq 2 \varepsilon \\ k_{2}\left[L_{i}-(1+\varepsilon) L_{0 i}\right] ; & \varepsilon_{i}<2 \varepsilon\end{cases}
$$

Where $F$ is the force exerted, $k_{1}$ and $k_{2}$ are the spring stiffness coefficients, $\varepsilon_{i}$ is the engineering strain, and $L$ and $L_{0}$ are the spring current and slack lengths of the ligaments, respectively. Stiffness coefficient for each ligament was adopted from previous studies [27]. Quadriceps, patellar and gastrocnemius tendons were modeled from fully elastic solid elements with elastic modulus of
$200 \mathrm{MPa}, 363 \mathrm{MPa}$ and $1200 \mathrm{MPa}$, respectively [28]-[29] . Figure 1 shows the degree-of-freedom of the knee simulator. The pelvis could displace up and down and the ankle joint was allowed to translate in mediolateral axis to simulate adduction-abduction motion and to rotate in all axis. Therefore, the knee joint performed six-degree-of-freedom motions.

The quadriceps were actuated from 0 to peak force of $200 \mathrm{~N}$ [22] while gastrocnemius muscles were loaded from 0 to $1700 \mathrm{~N}$ [21] within 0.2 seconds from extension position. High rate of muscle loading was intentionally applied to create inertial effects during squatting motion without compromising the knee stability. Gradual muscle forces were initially applied to induce compressive load at the knee joint which allowed the femoral component to resolve into neutral position before knee flexed, as well as to avoid instability in computational process. Hamstrings muscles were simulated as three active springs consist of biceps femoris, semimembranosus and semitendinosus. The spring was modeled based on Hill's muscle model with activation developed within LS-Dyna environment. Peak isometric force for each muscle bundle was set to adapt previous study [30].

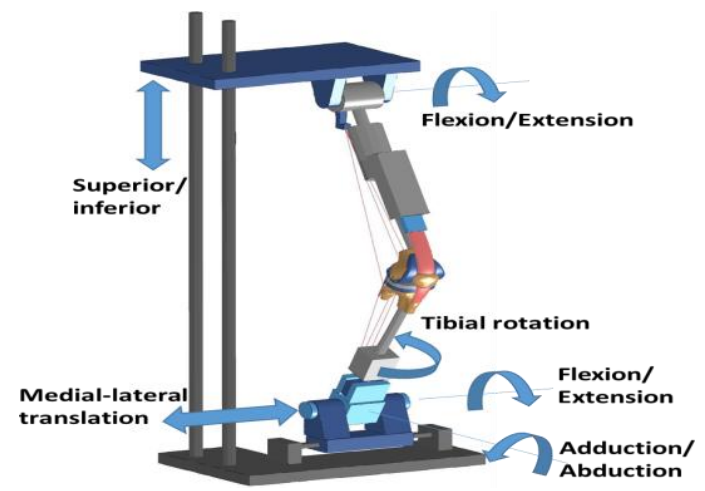

Fig. 1: Degree-of-freedom of the computational knee simulator

\section{Results}

FE model of the knee prosthesis was previously validated in prior study [31]. Kinematics verification of the knee simulator model was carried out by comparing the axial tibial rotation with in vivo analysis on the same design of prosthesis, by Shi et al. [1]. A good agreement was shown whereas internal tibial rotation achieved by Superflex was $5.3^{\circ}$ from knee simulator FE model and $5.1^{\circ} \pm 1.8^{\circ}$ from in vivo analysis. Figure 2 illustrates the internal tibial rotation of Superflex and NRG during knee bending motion. The tibial rotation of NRG increased tremendously, while gradual increase was shown by Superflex after post-cam engagement occurred.

Figure 3 shows the maximum von Mises stress as the function of flexion angle at PE tibial post. A sudden increase in stress at tibial post was observed at $82^{\circ}$ for Superflex and $63^{\circ}$ for NRG, showing that the post-cam engagement occurred at those flexion angles. The peak values of maximum von Mises stress at tibial post were $80 \mathrm{MPa}$ and $50 \mathrm{MPa}$ for Superflex and NRG, respectively.

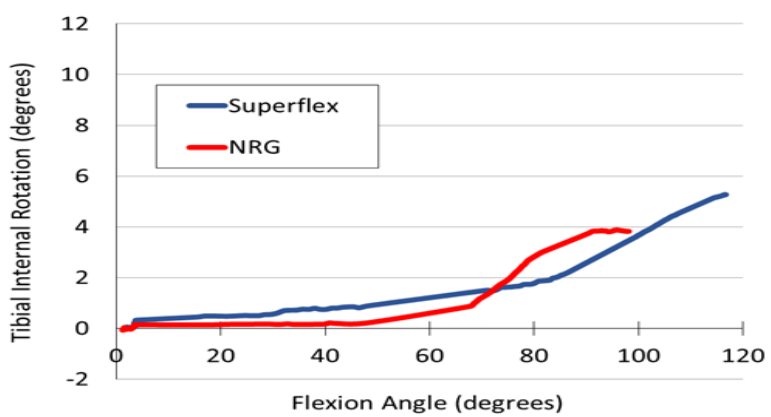

Fig. 2: The variations of internal tibial rotation of Superflex and NRG with knee flexion angle. 


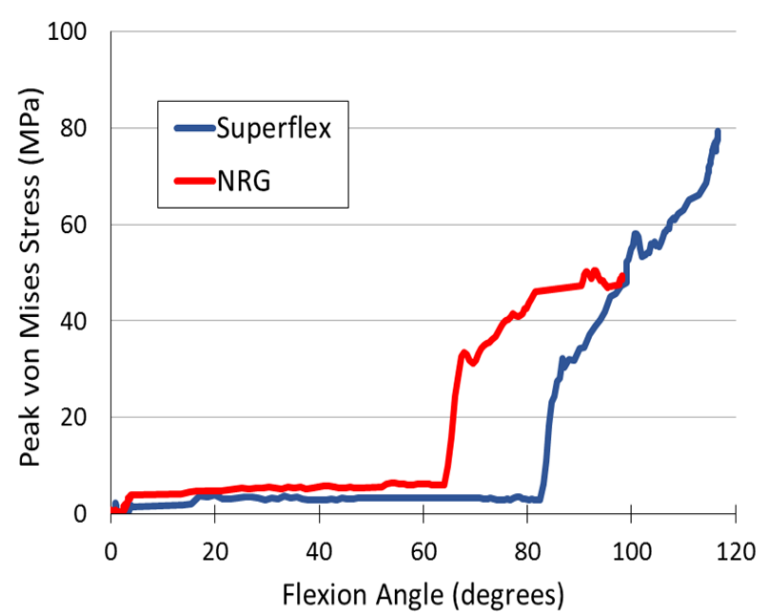

Fig. 3: The variations of internal tibial rotation of Superflex and NRG with knee flexion angle.

\section{Discussion}

Ligaments surround the knee joint are the key contributor to knee stability. Posterior cruciate ligament (PCL) is one of the major knee stabilizer that avoid posterior subluxation of tibial bone. It is typically resected during total knee replacement (TKR) especially in the case of degenerative PCL. Introduced in the last four decades, posterior-stabilized (PS) TKR procedure was designed to substitute PCL function in governing anterior-posterior (AP) translation and allowing femoral rollback [1]. PS-TKR knee implant is commonly designed to have a tibial spine and femoral cam that interact at certain flexion angle as the knee bends.

For Asians especially in Japan, East Asia and Middle East countries, squatting is one of the most common activities, such as seiza on tatami, kneeling and squatting during prayer. Prior studies revealed substantial normal and shear load at knee joint during such motion. More than 5 times body weight of normal force at knee joint was computed during descending to squat position [16]. Nagura et al. implemented inverse dynamics approach to assess knee kinetics from kinematics data of double leg squatting and obtained compressive and shear forces of $60 \%$ and $50 \%$ of bodyweight, respectively [46]. In vivo assessment which typically involved fluoroscopic analysis found that tibial rotation varied accordingly with implant geometry [31], [32], [43]-[44], [47]-[48]. Hence, the combination of such kinematics and kinetics of knee joint during high flexion angle is anticipated to be the cause of tibial post wear and damage.

In present study, the results have shown that tibial post engaged femoral cam at $82^{\circ}$ and $63^{\circ}$ of flexion angle for Superflex and NRG, respectively, in agreement with previous studies by Fitzpatrick et al. and Arnout et al. [34]-[35]. Early post-cam engagement of NRG was due to larger tibial post and shorter initial post-cam distance in comparison to Superflex. Both designs performed internal tibial rotation. This observation is attributed by larger isometric force of medial hamstrings and greater lateral laxity of knee joint in axial rotation. Insertion point of LCL has larger distance to axis of rotation compared to MCL, creates an effective moment arm during the action of hamstring muscles. Thus, medial compartment performs greater posterior translation results in internal rotation of the tibia. This conforms prior investigations by Kwak et al. and Victor et al. [36]-[37]. Larger internal rotation, however, was obtained by NRG even though both implants have curve-oncurve post-cam geometries. Von Mises stress of Superflex increased tremendously from $82^{\circ}$ to maximum flexion angle due to the edge loading sustained by significantly small radius at posteromedial surface of tibial post. This is in agreement with experimental study by Nakayama et al, found that the peak contact stress of Superflex tibial post substantially increased with internal rotation at $90^{\circ}$ of flexion [4]. This post-cam geometry of Superflex also has contributed to its limited tibial rotation in comparison to NRG.

Our study demonstrated the practicality of computational model to anticipate tibial rotation, post-cam engagement flexion angle, engagement velocity and prosthesis internal stress. The knee simulator ligamentous constraints were appropriately implemented based on previous experimentally validated soft tissue model [27]. Decent kinematic behavior replicated the corresponding prosthesis design measured in prior in vivo studies was clearly exhibited. However, there are some limitations of this investigation. The knee simulator performed purely muscle force driven motion where control scheme was not implemented to regulate the muscle load in reproducing corresponded flexion angle and joint load from experimentally measured data of knee squat. To the best of our knowledge, there is no in vivo joint load acquired from TKA patients performing rapid squat currently available due to limited number of young and active patients. Analysis was only performed for squat activity. This activity, however, was selected as it provided adequate post-cam interaction for analysis. The simulation only compared a very limited number of knee prosthesis designs. More prosthesis designs provide better correlation analysis between different geometrical features. Both implant models were chosen as NRG is the improved design of Superflex, hence the impact of geometrical design improvement could be verified.

\section{Conclusion}

In conclusion, the developed computational knee simulator has successfully assessed the impact of geometry on the post-cam engagement mechanics associated with the knee kinematics and the stress distribution at tibial post of TKA prostheses during dynamic activity. The knowledge from the present study may beneficial for future implant design that facilitate better knee kinematics and improve the implant survivorship through postcam geometrical features.

\section{Acknowledgement}

This work was funded by Universiti Teknologi MARA (UiTM) under Bestari Perdana Research Grant (600-IRMI/DANA 5/3 BESTARI (P) (058/2018).

\section{References}

[1] X. Shi, Z. Zhou, B. Shen, J. Yang, P. Kang, and F. Pei, "Variations in morphological characteristics of prostheses for total knee arthroplasty leading to kinematic differences," The Knee, vol. 22, no. 1, pp. 18-23, Jan. 2015.

[2] M. A. Baldwin, C. W. Clary, C. K. Fitzpatrick, J. S. Deacy, L. P. Maletsky, and P. J. Rullkoetter, "Dynamic finite element knee simulation for evaluation of knee replacement mechanics," $J$. Biomech., vol. 45, no. 3, pp. 474-483, Feb. 2012.

[3] M. Tamaki, T. Tomita, T. Yamazaki, W. J. Hozack, H. Yoshikawa, and K. Sugamoto, "In Vivo Kinematic Analysis of a High-Flexion Posterior Stabilized Fixed-Bearing Knee Prosthesis in Deep KneeBending Motion," J. Arthroplasty, vol. 23, no. 6, pp. 879-885, Sep. 2008 .

[4] K. Nakayama, S. Matsuda, H. Miura, H. Higaki, K. Otsuka, and Y. Iwamoto, "Contact stress at the post-cam mechanism in posteriorstabilised total knee arthroplasty," J. Bone Joint Surg. Br., vol. 87B, no. 4, pp. 483-488, Apr. 2005.

[5] Y. Akasaki, S. Matsuda, T. Shimoto, H. Miura, H. Higaki, and Y. Iwamoto, "Contact Stress Analysis of the Conforming Post-Cam Mechanism in Posterior-Stabilized Total Knee Arthroplasty," J. Arthroplasty, vol. 23, no. 5, pp. 736-743, Aug. 2008.

[6] C.-H. Huang, J.-J. Liau, C.-H. Huang, and C.-K. Cheng, "Stress analysis of the anterior tibial post in posterior stabilized knee prostheses," J. Orthop. Res., vol. 25, no. 4, pp. 442-449, Apr. 2007.

[7] C.-H. Huang, J.-J. Liau, C.-H. Huang, and C.-K. Cheng, "Influence of Post-cam Design on Stresses on Posterior-stabilized Tibial Posts," Clin. Orthop., vol. 450, pp. 150-156, Sep. 2006. 
[8] V. Digennaro, F. Zambianchi, A. Marcovigi, R. Mugnai, F. Fiacchi, and F. Catani, "Design and kinematics in total knee arthroplasty," Int. Orthop., pp. 1-7.

[9] H. Pandit, T. Ward, D. Hollinghurst, D. J. Beard, H. S. Gill, N. P. Thomas, and D. W. Murray, "Influence of surface geometry and the cam-post mechanism on the kinematics of total knee replacement," J. Bone Joint Surg. Br., vol. 87-B, no. 7, pp. 940-945, Jul. 2005.

[10] Y.-S. Chiu, W.-M. Chen, C.-K. Huang, C.-C. Chiang, and T.-H Chen, "Fracture of the polyethylene tibial post in a NexGen posterior-stabilized knee prosthesis," J. Arthroplasty, vol. 19, no. 8, pp. 1045-1049, Dec. 2004.

[11] P. Mestha, Y. Shenava, and J. C. D'Arcy, "Fracture of the polyethylene tibial post in posterior stabilized (Insall Burstein II) total knee arthroplasty," J. Arthroplasty, vol. 15, no. 6, pp. 814-815, Sep. 2000.

[12] H. D. Clarke, K. R. Math, and G. R. Scuderi, "Polyethylene post failure in posterior stabilized total knee arthroplasty," $J$. Arthroplasty, vol. 19, no. 5, pp. 652-657, Aug. 2004.

[13] I. C. Burgess, M. Kolar, J. L. Cunningham, and A. Unsworth, "Development of a six station knee wear simulator and preliminary wear results," Proc. Inst. Mech. Eng. [H], vol. 211, no. 1, pp. 3747, Jan. 1997.

[14] O. K. Muratoglu, C. R. Bragdon, M. Jasty, D. O. O’Connor, R. S. Von Knoch, and W. H. Harris, "Knee-simulator testing of conventional and cross-linked polyethylene tibial inserts," $J$. Arthroplasty, vol. 19, no. 7, pp. 887-897, Oct. 2004.

[15] J. D. DesJardins, P. S. Walker, H. Haider, and J. Perry, "The use of a force-controlled dynamic knee simulator to quantify the mechanical performance of total knee replacement designs during functional activity," J. Biomech., vol. 33, no. 10, pp. 1231-1242, Oct. 2000.

[16] L. P. Maletsky and B. M. Hillberry, "Simulating Dynamic Activities Using a Five-Axis Knee Simulator," J. Biomech. Eng., vol. 127, no. 1, pp. 123-133, Mar. 2005.

[17] J. P. Halloran, C. W. Clary, M. Taylor, A. J. Petrella, P. J. Rullkoetter, and L. P. Maletsky, "Verification of Predicted Knee Replacement Kinematics During Simulated Gait in the Kansas Knee Simulator," J. Biomech. Eng., vol. 132, no. 8, pp. 081010 081010, Jul. 2010.

[18] A. C. Godest, M. Beaugonin, E. Haug, M. Taylor, and P. J. Gregson, "Simulation of a knee joint replacement during a gait cycle using explicit finite element analysis," J. Biomech., vol. 35 , no. 2, pp. 267-275, Feb. 2002.

[19] R. F. Escamilla, "Knee Biomechanics of the Dynamic Squat Exercise," Med. Sci. Sports Exerc., vol. 33(1), pp. 127-141, Jan. 2001.

[20] N. J. Dahlkvist, P. Mayo, and B. B. Seedhom, "Forces during Squatting and Rising from a Deep Squat," Eng. Med., vol. 11, no. 2, pp. 69-76, Apr. 1982.

[21] T. Finni, P. V. Komi, and V. Lepola, "In vivo human triceps surae and quadriceps femoris muscle function in a squat jump and counter movement jump," Eur. J. Appl. Physiol., vol. 83, no. 4-5, pp. 416-426, Nov. 2000.

[22] G. Li, T. W. Rudy, M. Sakane, A. Kanamori, C. B. Ma, and S. L.-Y. Woo, "The importance of quadriceps and hamstring muscle loading on knee kinematics and in-situ forces in the ACL," J. Biomech., vol. 32, no. 4, pp. 395-400, Apr. 1999.

[23] Y. Yonei, Y. Miwa, S. Hibino, Y. Takahashi, R. Miyazaki, T. Yoshikawa, H. Moriwaki, T. Hasegawa, T. Hiraishi, and K. Torii, "Japanese Anthropometric Reference Data - Special Emphasis on Bioelectrical Impedance Analysis of Muscle Mass," Anti-Aging Med., vol. 5, no. 6, pp. 63-72, 2008.

[24] D. Winter A., Biomechanics and Motor Control of Human Movement, 4th ed. Wiley, 2009.

[25] S. Hall J., Basic Biomechanics, 6th ed. New York: McGraw Hill, 2012.

[26] J. P. Halloran, A. J. Petrella, and P. J. Rullkoetter, "Explicit finite element modeling of total knee replacement mechanics," $J$. Biomech., vol. 38, no. 2, pp. 323-331, Feb. 2005.

[27] E. M. Abdel-Rahman and M. S. Hefzy, "Three-dimensional dynamic behaviour of the human knee joint under impact loading," Med. Eng. Phys., vol. 20, no. 4, pp. 276-290, Jun. 1998.

[28] H. U. Stäubli, L. Schatzmann, P. Brunner, L. Rincón, and L.-P Nolte, "Mechanical Tensile Properties of the Quadriceps Tendon and Patellar Ligament in Young Adults," Am. J. Sports Med., vol. 27, no. 1, pp. 27-34, Jan. 1999.
[29] C. N. Maganaris and J. P. Paul, "Tensile properties of the in vivo human gastrocnemius tendon," J. Biomech., vol. 35, no. 12, pp. 1639-1646, Dec. 2002.

[30] E. M. Arnold, S. R. Ward, R. L. Lieber, and S. L. Delp, "A Model of the Lower Limb for Analysis of Human Movement," Ann. Biomed. Eng., vol. 38, no. 2, pp. 269-279, Dec. 2009.

[31] M. A. Mohd Anuar, M. Todo, R. Nagamine, and S. Hirokawa, "Dynamic Finite Element Analysis of Mobile Bearing Type Knee Prosthesis under Deep Flexional Motion," Sci. World J., vol. 2014, p. e586921, Jul. 2014.

[32] T. Nagura, C. O. Dyrby, E. J. Alexander, and T. P. Andriacchi, "Mechanical loads at the knee joint during deep flexion," J. Orthop. Res., vol. 20, no. 4, pp. 881-886, 2002.

[33] K. Kanekasu, S. A. Banks, S. Honjo, O. Nakata, and H. Kato, "Fluoroscopic analysis of knee arthroplasty kinematics during deep flexion kneeling," J. Arthroplasty, vol. 19, no. 8, pp. 998-1003, Dec. 2004.

[34] C. K. Fitzpatrick, C. W. Clary, A. J. Cyr, L. P. Maletsky, and P. J. Rullkoetter, "Mechanics of post-cam engagement during simulated dynamic activity," J. Orthop. Res., vol. 31, no. 9, pp. 1438-1446, Sep. 2013.

[35] N. Arnout, L. Vanlommel, J. Vanlommel, J. P. Luyckx, L. Labey, B Innocenti, J. Victor, and J. Bellemans, "Post-cam mechanics and tibiofemoral kinematics: a dynamic in vitro analysis of eight posterior-stabilized total knee designs," Knee Surg. Sports Traumatol. Arthrosc., pp. 1-11, Jul. 2014.

[36] S. D. Kwak, C. S. Ahmad, T. R. Gardner, R. P. Grelsamer, J. H. Henry, L. Blankevoort, G. A. Ateshian, and V. C. Mow, "Hamstrings and iliotibial band forces affect knee kinematics and contact pattern," J. Orthop. Res., vol. 18, no. 1, pp. 101-108, Jan. 2000 .

[37] J. Victor, L. Labey, P. Wong, B. Innocenti, and J. Bellemans, "The influence of muscle load on tibiofemoral knee kinematics," $J$. Orthop. Res., vol. 28, no. 4, pp. 419-428, 2010 